\title{
SAR Target Detection Method Based On Empirical Mode Decomposition
}

\author{
Shiqi Huang ${ }^{1,}$, Beihe Wang ${ }^{1}$, Yihong $\mathrm{Li}^{1}$ and Bei Ge ${ }^{1}$ \\ ${ }^{1}$ Xi'an Research Institute of Hi-Tech, Hongqing Town, 710025, Xi'an, P. R. China \\ aE-mail: hshiqi@sina.com
}

Keywords: Empirical mode decomposition; SAR image; Target detection

\begin{abstract}
Empirical mode decomposition (EMD) is a new signal processing theory, and it is very much fitting for non-stationary signal processing, such as radar signal. So this paper proposes the new synthetic aperture radar (SAR) image target detection algorithm after analyzing the characteristics of EMD and SAR images. The proposed method performs the EMD operation, feature extraction, election and fusion, which can reduce the affection of speckle. Experimental results show that the proposed method is very effective.
\end{abstract}

\section{Introduction}

Synthetic aperture radar is an active microwave imaging radar, and the unique characteristic of it is that can obtain data under all-time and all-weather. Therefore, SAR is widely applied in many fields, such as disaster monitoring, ocean and environment monitoring, military information reconnaissance $[1,2]$. SAR imaging receives the backscattering information of ground object, and then performs the complex operation processing with some imaging algorithm and the SAR image of describing feature image space of the ground object is obtained. The backscattering signal is the typical non-linearity and non-stationary signal. Although wavelet transform can process non-linearity and non-stationary signal, it need the subjective choice for the basis function and it has great relation with the prior experimental knowledge, so it affects and limits the applications of wavelet transform. EMD is a new and complete data-driven processing method and it need not choose the basis function and perform the self-adaptation adjustment according with the signal itself characteristics. So EMD overcomes the inherent limitation of wavelet transform and has obvious advantage in non-linearity and non-stationary signal processing. Therefore, this paper proposes a new SAR target detection algorithm based on EMD.

\section{Empirical mode decomposition theory}

The correlation theory and conception about EMD are proposed by Huang of American NASA in 1998 [3]. After a signal is decomposed by EMD theory, a intrinsic mode function (IMF) and a residue can be obtained. Then the continued decomposition goes on with the residue, and the new IMF and residue can be obtained. The operation does not stop until it satisfied some conditions. Wavelet multi-scale analysis theory realize the scale adjustments via choosing different wavelet basis function, and these basis functions are mutual independence, furthermore, they have none any connection with the concrete signal. Therefore, it is very easy to make some detailed information to be omitted, especially, image information processing. EMD does not choose the basis function which is produced by signal itself, furthermore, different signal has different basis function, which not only is unique, but also need not choose. So EMD has been applied widely in non-linearity and non-stationary signal processing, such as speech signal, earthquake signal, water wave signal $[4,5]$.

EMD is a new time-frequency analysis method for non-linearity and non-stationary signal processing, and is extended two-dimension signal processing [6]. At first, two-dimension signal is decomposed into one-dimension according to row or column, and then is be processed with EMD. This way is fitting for extracting level and vertical direction information, but it omits the spatial 
correlation of two-dimension signal. Therefore, some scholars proposed the bidimensional empirical mode decomposition (BEMD), and two-dimension signal can be processed directly with BEMD. This promotes the applications of EMD in two-dimension signal, such as image processing [7].

The decomposed idea of BEMD is as same as EMD. It decomposes a complex two-dimension signal into some bidimensional intrinsic mode function (BIMF) and a residue. Every BIMF is independent each other, and they respectively local feature signals which include different frequency and different space of original signal.

The sieving procedure is introduced for BEMD with two-dimension image signal. Let the processed image is $I(x, y)$, the envelope curve surface of the maximum value is $E_{\max }(x, y)$, the envelope curve surface of the minimal value is $E_{\min }(x, y)$. The realized step is as following.

(1) Seeking the local maximum value and minimal value on the curve surface of an image $I(x, y)$;

(2) Using the interpolative method to perform interpolation operation on extremal points, and obtaining the envelope curve surface of $E_{\max }(x, y)$ and $E_{\min }(x, y)$;

(3) Computing the mean envelope curve surface, i.e. $E_{\text {mean }}(x, y)=\left[E_{\max }(x, y)+E_{\min }(x, y)\right] / 2$, and extracting high frequency detailed information, i.e. $d(x, y)=I(x, y)-E_{\text {mean }}(x, y)$;

(4) Judging the two conditions of BIMF whether or not $d(x, y)$ satisfies. If it does not satisfy them, then $I(x, y)=d(x, y)$, and the repeat operations are performed from the step one to the step four. Otherwise, $d(x, y)$ is taken as the first BIMF component, i.e. $\operatorname{bimf}_{1}(x, y)$;

(5) Let $r_{1}(x, y)=I(x, y)-\operatorname{bimf}_{1}(x, y)$, and it is taken as a new signal that will be decomposed. Repeating the step one to the step four, then the second BIMF is obtained, i.e. $\operatorname{bimf}_{2}(x, y)$. The residue is $r_{2}(x, y)=R_{1}(x, y)-\operatorname{bimf}_{2}(x, y)$. The forementioned course is repeated until the residue only has one maxima and none BIMF component can be extracted.

Finally, the image $I(x, y)$ can be expressed by the sum of some BIMF components and the residuce, and it is given by

$$
I(x, y)=\sum_{i=1}^{M} \operatorname{bimf}_{i}(x, y)+R_{M}(x, y)
$$

Where $M$ is the number of BIMF components, and $R_{M}(x, y)$ denotes the residuary component.

In step (4), judging whether or not finishing of the sieving process, it need see whether they satisfy the two conditions that the definition of BIMF function contains. It usually used the standard deviation between the adjioning two time sieving results to judge the condition, and this is given by

$$
S D=\sum_{x=0}^{K} \sum_{y=0}^{L}\left[\frac{\left|d_{i-1}(x, y)-d_{i}(x, y)\right|^{2}}{d_{i-1}^{2}(x, y)}\right]
$$

Where $K$ and $L$ denote the ordinal number of the EMD decomposition, and the value of $S D$ is often from 0.2 to 0.3 .

\section{The description of SAR target detection algorithm}

EMD uses the self feature of signal to decompose the signal, therefore, not only it is a full data-driven method for signal processing, but also it is an adaptive, multi-scale and multi-resolution analysis method. SAR echo signal is non-stationary signal. If it is decomposed with EMD, more accurate target information can be obtained, which is very much fitting for target detection, classification and recognition. The flow chart of the new target detection algorithm based on EMD is shown in Fig.1.

The proposed algorithm for SAR image target detection mainly includes steps as follow.

(1) Input SAR images. These SAR images are processed through the geometry correction and radiometric correction. 
(2) Make multi-scale decomposition operation with EMD or BEMD. When an SAR image is decomposed, two ways can realize the operations. One is that the SAR image is directly decomposed by EMD and then the characteristics are extracted. In fact, when an two-dimension image are

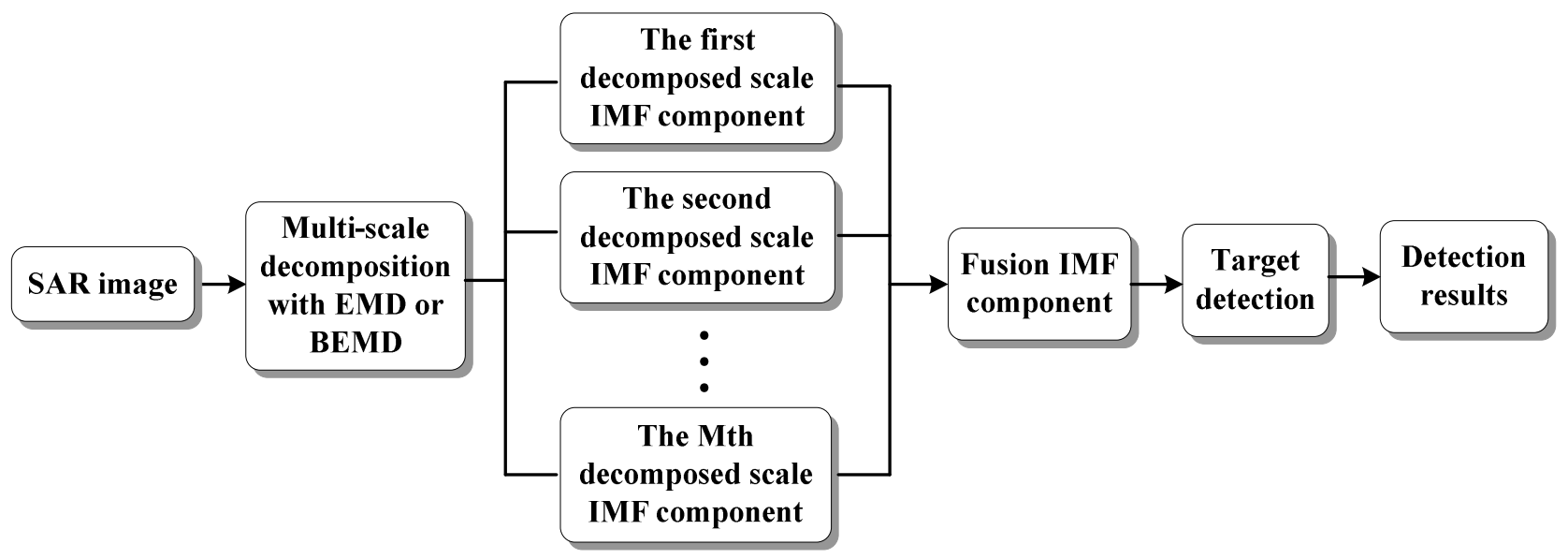

Fig.1 The flow chart of SAR image target detection algorithm

processed by the method, every row or every column of the image is acted as one vector signal, and then the row vector or the column vector performs one-dimension EMD. The other is that the SAR image is directly decomposed and characteristics are extracted by BEMD.

(3) Obtain all decomposed scale IMF component.

(4) Select IMF component and fuse them.

(5) Perform target detection operation with the fused IMF characteristic image.

(6) Obtain the detection results of SAR targets.

\section{Experimental results and analysis}

At present, the widely applied target detection method is the constant false alarm ratio (CFAR) detection algorithm mainly. There are many branch direction so far, such as the cell-average CFAR (CA-CFAR), the smallest of CFAR (SO-CFAR), the order statistic CFAR (OS-CFAR) and the greatest of CFAR (GO-CFAR). The main difference of them is the different clutter mean estimation algorithms. In this experimentation, the CA-CFAR algorithm, the two-parameter CFAR (TP-CFAR) algorithm [8] and the coherence-based CFAR (CCFAR) algorithm [9] are used to test the new algorithm. The experimental data comes from the SAR image databank of the moving and stationary target acquisition and recognition (MSTAR), and they are shown in Fig.2. The Fig.2(A) is original image, and is the SAR image slice of a tank 72 in MSTAR databank. The Fig.2(B) and Fig.2(C) are the obtained BIMF image with EMD and BEMD, respectively. The Fig. 3 is the experimental results. The Fig.3(a) is the CCFAR algorithm, the Fig.3(b) is the CA-CFAR algorithm and the Fig.3(c) is the TP-CFAR algorithm. The false alarm ratio is $1 \mathrm{e}-8,1 \mathrm{e}-6$ and $1 \mathrm{e}-3$ in turn from the Fig.3(a) to the Fig.3(c).

It is known from the Fig. 2 that the different method can obtain different information, so the BIMF characteristics are different via them, but there are more abundant characteristic information in two dimension EMD than one dimension EMD. Under anything conditions, even though the same CFAR condition, the obtained results with BEMD are better than that with EMD, which is shown in Fig.3. Especially, when they combine with the CCFAR detection method, the effect is more obvious. 

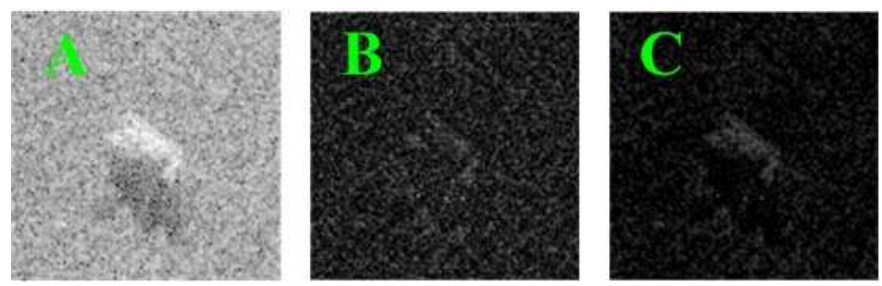

Fig.2 The original SAR image and BIMF image
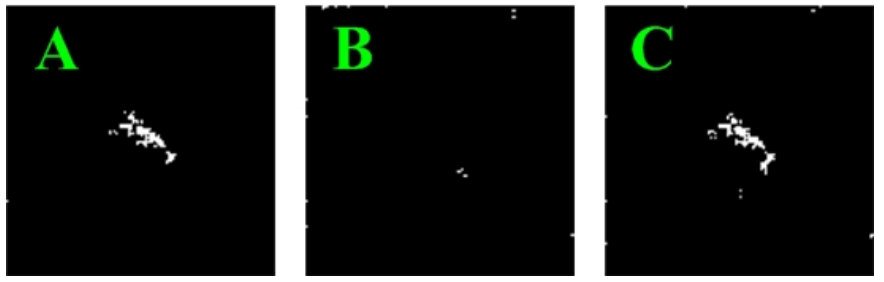

(a) The CCFAR algorithm
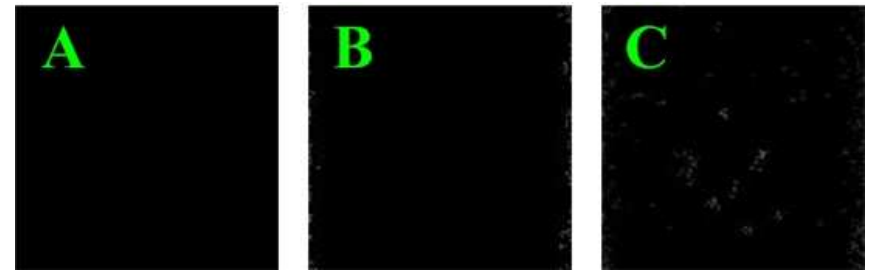

(b) The CA-CFAR algorithm
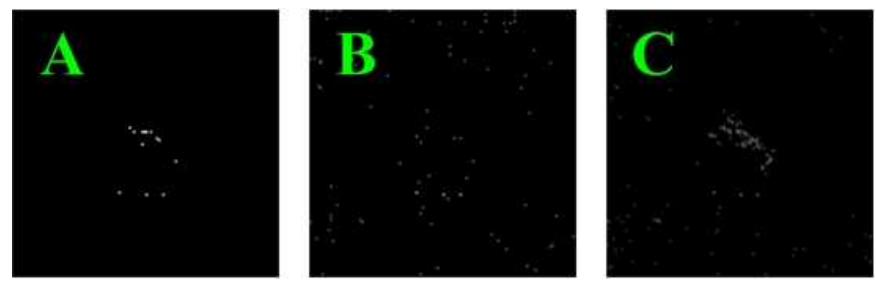

(c) The TP-CFAR algorithm

Fig.3 The experimental results

\section{Conclusions}

This paper studies the EMD theory and SAR image data characteristic, and then proposes a new SAR target detection algorithm. The experimental results show that the proposed method is very much effective, especially, when it connects the CCFAR detection method, the detection effect is better.

\section{Acknowledgements}

This work has been supported by China Postdoctoral Science Foundation (No. 20100471767), China Postdoctoral Special Science Foundation (No. 2011004751) and Shanxi Province National Natural Science Foundation (No.2011JM5006). 


\section{References}

[1] P. Matgen, R. Hostache, G. Schumann, L. Pfister, L. Hoffmann, H.H.G. Savenije. Towards an automated SAR-based flood monitoring system: Lessons learned from two case studies. Physics and Chemistry of the Earth. 36 (2011): 241-252.

[2] H. J.You. SAR change detection by multi scale segmentation and optimization. Geomatics and Information Science of Wuhan University in Chinese, 36(5)(2011): 531-534.

[3] E. N. Huang, et al. The empirical mode decomposition and the Hilbert spectrum for nonlinear and non-stationary time series analysis[A].Proc. R. Soc. London. A454 (1998), 903-995.

[4] H. Ling, L. Margaret, C. M. Namunu, B. A. Nicholas. Study of empirical mode decomposition and spectral analysis for stress and emotion classification in natural speech. Biomedical Signal Processing and Control. 6(2)(2011): 139-146.

[5] B. V. Manuel, W. Binwei, E. B. Kenneth. ECG signal denoising and baseline wander correction based on the empirical mode decomposition. Computers in Biology and Medicine. 38(1)(2008): $1-13$.

[6] J. L. Sancheza, J. J. Trujillob. Improving the empirical mode decomposition method. Applicable Analysis, 90(3-4)(2011): 689-713.

[7] G. L. Xu, X. T. Wang, X. G. Xu. Improved bi-dimensional empirical mode decomposition based on 2d-assisted signals: analysis and application, IET Image Processing. 5(3)(2011): 205-221.

[8] L. M. Novak, G. J. Owirka and C. M. Netishen. Performance of a High-resolution Polarimetric SAR Automatic Target Recognition System. Lincoln Laboratory Journal. 6(1)(1993): 11-23.

[9] S. Q. Huang, D. Z. Liu, G. Q. Gao, X. J. Guo. A novel method for speckle noise reduction and ship target detection in SAR images. Pattern Recognition. 42(7) (2009): 1533-1542. 